pyloric obstruction or ulcer of the stomach as disclosed by autopsy findings, and of stasis of any kind, as disclosed by clinical investigation. The conception of a connection between the two conditions in the other direction, the long standing hypersecretion serving as a contributing cause to the cirrhosis, has, as far as I know, no facts of medical observation to support it. It is not suggested by the history of this case.

The second case which I have to report is one of intermittent or periodic hypersecretion. The existence of cirrhosis in this case is based upon the finding of an enlarged liver combined with the alcoholic history of the patient. The patient is still alive. The record of the case is as follows:

The patient, a bartender, age forty-four, was first seen by me in February, 1905. For about a year previous to this time he had been having acute attacks of stomach trouble, alternating with periods of well being. The symptoms of the attacks were as follows: Following a period of well being the patient would be seized with severe pain in the stomach, followed by vomiting. Following this for a period of one to three weeks there would be constant nausea with frequent vomiting, distaste for food, excessive thirst. The vomiting, as a rule, followed the ingestion of food in an hour or so, but some times came in the early morning or long after eating.

The vomitus consisted of large amounts, was watery and acid. It had never contained blood. At the start of the attacks the pain was very acute and relieved temporarily by the vomiting. The pain was at the pit of the stomach and beneath the sternum, never in the right side. There had been no jaundice. The first attack which occurred lasted one week. The patient was then perfectly well for three months, and then had a second attack lasting two weeks. Following this there was a period of well being lasting two months and then the present attack. There was loss of flesh during an attack and a reestablishment in the intervals. The patient had been a steady drinker. No special occasion for the advent of the attacks could be discovered. He was frequently drunk during the intervals of well being. He denied syphilis. The condition of the patient at the time of my visit was as follows:

Well developed; color, good; hemoglobin, $100 \%$; temperature normal; pulse 80 ; heart and lungs normal; abdomen full; no ascites; no enlargement of veins of abdominal wall. Liver dullness extended from sixth rib nipple line to two inches below costal border. Edge palpable and symmetrical. Spleen not palpable. Arteries were normal to the touch. Reflexes normal.

The contents of the stomach, expressed in the morning after a twelve hours' fast, had the following characteristics:

Quantity, 500 cc. Character, watery with very little sediment. No food was present. Free HCl present; quantity was $0.12 \%$. Total acidity, .14\% No lactic acid. No blood by guaiac test. Inflation of stomach normal.

The contents expressed, two hours after an Ewald meal, had the following character:

Quantity, $250 \mathrm{cc}$; Consistency purely liquid. No food was present. Free $\mathrm{HCl}, 0.22 \%$. Total acidity, $.28 \%$.

The attack of stomach disturbance in which I saw the patient lasted three weeks. It was followed by a period of comparative well being. During the latter period the patient suffered from dyspeptic symptoms consisting of distress after eating, eructation of gas. There was no vomiting, and the patient considered himself as well as usual. The contents of the stomach obtained during this period expressed one hour after an Ewald meal, showed the following character:

Quantity, $150 \mathrm{cc}$. The proportion of supernatant fluid was rather greater than normal. Total free $\mathrm{HCl}$ was $.16 \%$.

Three months from the time of my first visit the patient had an acute attack similar to the one described lasting two weeks. Since then he has had two similar attacks. The size of the liver remains practically the same.

We have here a case of periodic or intermittent hypersecretion associated with liver cirrhosis. The cause of the attacks cannot be definitely determined. They may be due to acute irritation of the stomach from indulgence in alcohol. Local stomach causes, as stasis or ulcer of the stomach, can apparently be ruled out by a consideration of the results of clinical investigation. How far the cirrhosis is a contributing cause is a matter of surmise. The case, however, serves as a second example of the association of cirrhosis and gastric hypersecretion.

\section{THE SERUM TREATMENT OF EXOPHTHALMIC GOITRE.}

\author{
BY G. A. WATERMaN, M.D.,
}

Assistant in Neurology, Harvard Medical School; Assistant Visiting Physician to the Out-Patient Neurological Department, Massachusetts General Hospital.

Since the first recognition of exophthalmic goitre as a distinct disease by Parry in 1825, and later by Graves and Basedow, various theories as to the cause of the condition have been advanced.

The symptoms were early attributed to pressure on the cervical vessels and nerves by the enlarged gland. Later, the theory of disease of the cervical sympathetic was advanced. On account of emotional upsets being a frequent cause of the disease, many considered it to be a functional disorder of the nervous system, allied to hysteria. Blake, Thompson and others have advanced the idea that the symptoms are due to auto-intoxication from the alimentary canal, while others have maintained that the cause of the disease lies in a disturbance of the medulla.

The generally accepted theory at the present day, however, and the one upheld by Moebius, is that the symptoms of the disease are caused by excessive secretion of the thyroid gland. In favor of this hypothesis is the evidence that, (1) in most cases of exophthalmic goitre the thyroid gland is enlarged; (2) that partial excision of the gland in patients suffering from the disease so often results in a cure and (3) that the administration of thyroid in sufficiently large doses to healthy individuals may give rise to most of the characteristic symptoms of the disease. In experimenting on animals, too, these symptoms have been artificially produced. Edmonds ${ }^{1}$ by treating dogs and monkeys, with 
thyroid, found that he could bring on exophthalmos, tachycardia, loss of weight and sweating, while Murray ${ }^{2}$ observed a similar result from feeding the gland to rabbits.

The methods of treatment for exophthalmic goitre have annually increased in number since the first recognition of the disease, and we constantly see cases reported which have been favorably influenced by some new remedy. Among the most popular may be mentioned arsenic, iodide of potash, bromide of quinine, phosphate of soda, and intestinal antiseptics. Electricity, in the form of faradism and galvanism, and the $\mathrm{x}$-ray, have been of undoubted value in the treatment of this disease, and partial extirpation of the gland, though a dangerous operation, has given rise to many cures.

Accepting, however, the theory of hyperthyroidization as the cause of exophthalmic goitre, the rational method of treatment would seem to be that of serum therapy, by which we could introduce a substance to neutralize the harmful products of excessive thyroid secretion, or by which we could make use of a cytotoxic serum which has a selective, destructive action on the secreting cells of the thyroid gland alone.

Following along these lines, Ballet and Enriquez ${ }^{3}$ in 1895 , at the Congress of Alienists and Neurologists at Bordeaux, published their results of the use of serum of thyroidectomized dogs, which was given in doses of 5 to 15 c.c. with beneficial results.

In 1899, Burghart published the results of his work with Blumenthal, in which they treated a case with the modified blood of a myxoedematous patient, with temporary improvement. In the same paper they described the use of liquid serum of thyroidectomized dogs in one case, and the dried serum in two cases, all three cases showing improvement.

In 1901 Moebius $^{5}$ described the use of serum of thyroidectomized sheep in the treatment of three cases with beneficial results. To this serum he gave the name of antithyroidin.

In 1902 Schultes ${ }^{6}$ reported a cure in a case treated by the same method.

In 1903 Moebius $^{7}$ reported two more cases treated by his antithyroidin with improvement and stated his preference for this method over the use of "Rodagen," which he had also used. At this time he also made known the use of the dried blood of thyroidectomized sheep.

In 1903 Lepine ${ }^{8}$ brought forward the theory that a true antitoxic serum, like the diphtheritic antitoxin, should be derived from animals immunized to large doses of thyroid gland. He fed to goats larger and larger doses of sheep's thyroids and found that their tolerance could be much increased, so that, eventually, a serum was obtained, which, on being injected into dogs, produced somnolence, slowing of the pulse, and increase in weight.

In 1904 Murray $^{2}$ gave his results from the use of this method. He fed to rabbits increasing doses of a thyroid preparation for a month and used the serum from these animals in the treatment of two cases of exophthalmic goitre. The results, however, were disappointing, although some improvement was manifest.

In 1903 Adam $^{\circ}$ reported a case successfully treated with antithyroidin, in which the symptoms returned when the treatment was stopped but again disappeared on the readministration of the serum. Rosenfeld ${ }^{10}$ also reported a case treated about this time, but did not state the degree of improvement. Saniton and Pisante,11 in 1904, described three cases treated first with blood and then with serum of thyroidectomized sheep, one attended with a permanent cure and two with amelioration of symptoms. In the same year Leyden ${ }^{12}$ reported good results from the use of serum and blood of thyroidectomized sheep but stated that he did not consider this method superior to the use of "Rodagen." At this time Josionek ${ }^{13}$ also reported two cases treated by the serum given by the mouth. Both patients were improved but one more than the other, and Josionek considered this due to the fact that treatment was instituted earlier in the disease.

In $1905 \mathrm{Kuh}^{15}$ described the cases of eleven patients treated with the serum of thyroidectomized sheep, all of whom were improved in varying degrees; and later Mix ${ }^{14}$ reported excellent results in two cases. Later, in 1905, Hallion ${ }^{16}$ made known his preference for the use of the whole blood, preserved in glycerin and given in doses of one to two teaspoonfuls daily. He believed that the active principle lies more in the corpuscles than in the serum and stated that Enriquez had adopted this method, giving increasing doses so that three tablespoonfuls were eventually given in a day. Durig and Lomer ${ }^{17}$ have also recently each reported a case and Alexander ${ }^{17}$ reported three cases treated by the serum of thyroidectomized sheep, all of which were' benefited, but none cured.

The experience of the writer in the use of serum treatment of exophthalmic goitre is illustrated in the report of the following cases. The serum used in the treatment of these cases was derived from blood drawn from the jugular vein of the goat two weeks or more after the removal of the thyroid gland. It was invariably used subcutaneously.

CASE I is that of a girl eighteen years old. In the fall of 1902 she was subjected to an unpleasant experience which caused her considerable mental shock. A month later she began to notice that her collars were tight on account of a swelling in the throat, and about this time her friends called attention to the unnatural prominence of her eyes. At this time she suffered from insomnia, vomiting, palpitation, sweating and tremor. She was given the rest cure for three months, but without improvement. She then went into the country, where she improved to a certain point, but when seen by the writer she had been in about the same condition for a year. There was marked exophthalmos; the thyroid was symmetrically enlarged; the pulse varied from 130 to 150 , and there was a pronounced fine tremor of the hands and head. The 
patient was about her daily duties, but was nervous and easily tired. The serum treatment was begun in May, 1904, and for three months she had doses of 1 cc. given subcutaneously about five times a week. During this period the patient's symptoms steadily improved, and there was a gradual lessening of all the physical signs, so that the exophthalmos and tremor had practically disappeared. The thyroid was no longer visible and scarcely palpable and the pulse ranged about 120. During the next three months the serum was given in 2 cc. doses about twice a week with continued improvement in the patient's condition. There remained no trace of the tremor and thyroid enlargement and the eyes regained their natural expression, while the pulse ranged about 90. From this time she had 3 cc. of serum every eight or ten days for two months, and now for a year and a half has remained free from signs of the disease, and with a constant pulse of 70 to 75 .

CASE II is also that of a girl eighteen years of age She was first seen in June, 1905 . She had always been delicate in health and easily tired, but during the year previous to her treatment she had seemed unnaturally weak, so that a walk of two or three blocks fatigued her. She had first noticed an enlargement of the thyroid in March, 1905, and for this she had been treated by Dr. T. M. Rotch, who had given her the dried blood of thyroidectomized goats for three months, but without benefit. At the time serum treatment was begun, examination showed a slight degree of exophthalmos, moderate enlargement of the thyroid gland and a pulse of 100 . There was, however, no tremor and no special irritability, but she complained of sweating, backache, and a constant sense of fatigue. This patient was given daily doses of 2 cc. of serum for a week, with no apparent change in her condition, except that a general urticaria resulted. The treatment was then suspended for two weeks, at the end of which time she was given daily doses of $1 \frac{1}{2}$ cc. for a period of two weeks. This resulted in a diminution in the girth of the throat of $1 \frac{1}{4}$ in. The pulse was constant at 90 and the exophthalmos had disappeared. The serum was then given in 3 cc. doses once a week for two months, and once a month for three months. It is now six months since the treatment was stopped and the patient shows no signs of the disease, has gained in weight and feels better than she had felt since she can remember. She takes walks of three and four miles without special fatigue and is as well able to attend to her social duties as her sisters are.

CASE III is that of a girl who, since childhood, had always been sensitive and high strung and subject to seizures of hysterical laughter and crying from slight emotional upsets. For many years she had had an irritable heart, and palpitation had been a source of annoyance to her. She had also been troubled with excessive sweating for some years, but as it was only a year and a half before the serum treatment was begun that she applied to her physician, on account of a tremor of the hands which was so marked that in drinking she was obliged to hold the glass with both hands. It was at this time that her attention was first called to the prominence of her eyes and to her goitre. The serum treatment was begun in this case in June, 1905. At that time there was marked exophthalmos and tremor, moderate enlargement of the thyroid and a pulse rate varying from 140 to 155 . Doses of 2 cc. of serum were given four times a week for two months, with marked improvement of all her subjective symptoms, a diminution in the size of the thyroid, a drop in the pulse to 120 , a decrease in the tremor and a marked lessening in the prominence of one of the eyes. The treatment has been continued for eight months, being given every eight to ten days, and still keeps the patient in this improved and comfortable condition, though on several occasions when she has gone two or three weeks without the serum, she has felt more nervous and has suffered from palpitation. It is interesting to note in connection with the prolonged treatment in this case that the hair has become dry and brittle and is turning gray.

CASE IV is that of another young woman, twentysix years of age, who had always been and was still of a nervous and hysterical temperament. At the time the serum treatment was instituted she had suffered for two years from tremor and palpitation. Examination showed a moderate enlargement of the thyroid and exophthalmos. Her hands were tremulous and her pulse was 120 . She was given ten doses of 2 cc. of serum in the course of thirty days, which resulted in the disappearance of the thyroid enlargement, some slowing of the pulse and an improvement in the general symptoms. For various reasons the treatment was not continued beyond this, and the patient in the course of two months lapsed into her former condition.

CASE V is that of a woman forty-one years old, who, for fifteen years, had had goitre, exophthalmos, tremor, palpitation and sweating. She had always been able to keep about, but had suffered much of the time from nervousness and palpitation and occasional attacks of diarrhea which were difficult to check.

Examination at the time serum treatment was begun (in the winter of 1905) showed marked exophthalmos and goitre. The hands were moist and tremulous; the heart showed hypertrophy of the left ventricle, and a cardio-respiratory murmur was heard over the whole precordia; the pulse was 110 .

She was given the serum in 2 cc. doses twice a week for three weeks, and daily for seven days. At first there was a slowing of the pulse to 80 beats a minute, with a cessation of the attacks of palpitation which had previously come daily. The last few days, however, the pulse again rose to 100 and the palpitation returned. The treatment was not continued beyond this point.

The writer has also tried dried blood of thyroidectomized goats, given in doses of $5 \mathrm{gr}$., two or three times a day in a number of cases, but has never seen beneficial results from its use.

Of the five cases here reported, we may consider two as cured, two improved and one unimproved. This number alone is, of course, insufficient for estimating the value of this method of therapy, and we must remember that many methods of treatment advocated for this disease have given results fully as favorable in a few cases, but in the long run have proved disappointing.

Looking over all the cases that have been reported in which the serum treatment has been employed, one cannot help feeling that we have in it an agent which does influence the disease favorably, and may, with further modification, prove of more uniform value. It may be along this line or by the use of the cytotoxic serum, which has been evolved by several investigators, and so successfully used by Beebe and Rogers, ${ }^{13}$ as was recently reported in their admirable paper, that we are to find a specific means of relieving the distressing symptoms of a most troublesome disease. 
REFERENCES.

1 Brit. Med. Jour., Oct. 1905, p. 1077.

2 Lrit. Med. Jour,, Oct.

3 Congrés des Alien \& Neurol., Bordeaux, Aout, 1895.

4 Deut. Med. Woch., 1899, $\mathrm{g}_{\text {. }}$ 27.

s Neurol. Centrabl., 1901, No. 22.

Münch. Med. Woch., 1902, No. 20

7 Ibid., 1903, No. 4.

(yon: Med, 1903, Nov. 29.

10 unch. Med. Woch., 1903 , No. 9.

Deutsch. Med. Woch., 1903, No. 20.

Rev. Neurol., 1904, p. 1109

13 Die. Med. Woch., 1904, No. 37

14 Jour. Am. Med. Assoc., 1905, April 22.

15 Medicine, Detroit, 1905.

${ }_{16}$ Presse Médicale, Paris. Nov., 1905.

17 Münch. Med. Woch. May 2,1905.

18 Jour. Am. Med. Assoc., Feb. 17, 1906.

INTRODUCTION TO THE STUDY OF THE FUNDAMENTAL CAUSE OF SPLANCHN OPTOSIS-ABDOMINAL INCOMPETENCE: DEVELOPMENTAL FACTOR.

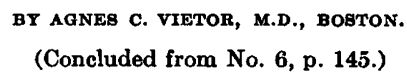

OUTLINE OF EVOLUTION OF SPECIFIC HUMAN ABDOMEN.

Correlated with evolution of pelvis, thorax and extremities; especially with extension, adduction and forward rotation of the lower extremities, so that the patella and toes point forward.

The final distinguishing characteristic of the human genus is the full extension of the lower extremities in the plane of the vertical trunk, the dorsal and ventral surfaces of which tend constantly to approach each other in approximately parallel planes.

This extension of the lower extremities upon the vertical trunk

(a) Removes the support of the erect (sitting) pelvis from the abdominal viscera.

(b) Removes the support of the flexed thighs which supplement the erect pelvis in supporting the abdomen.

(c) Adds forward rotation of the pelvis and anterior convexity of the lumbar spine; both of these conditions throwing the viscera forward and so facilitating their descent, directly through gravity and indirectly by increasing the stress on the supporting anterior and posterior abdominal walls, and so impairing the supporting power of these walls.

(d) Alters the conditions of respiratory equilibrium, because whatever affects the supporting power of the walls of the abdomen affects the action of the diaphragm, which latter is only another abdominal wall - the superior wall or roof.

If these various changes are not counterbalanced by compensatory changes in the anterior and posterior abdominal walls, they lead to further visceral displacement.

Soon after the enclosure of the viscera by the hody walls, the four extremities appear. Whether viewed as outgrowths of, or additions to, the body wall, these extremities are, throughout the vertebrate series, more or less bound to this body wall by muscles and integuments. This connection of the extremities with the body wall decreases as one rises in the animal series till in their highest development these extremities become specialized to nearly complete independence of the trunk; the latter serving only as a fixed point upon which all the more highly specialized movements of the extremities are based. This fixed point is utilized through the large muscles which pass from the trunk to the extremity. And these connecting muscles require a certain amount of trunk stability to furnish a point of departure for their activities.

In proportion as the evolution of the extremity is imperfect or incomplete, clinical observation shows that stress upon it is carried backward to its attachment to the trunk; and if this attachment is deficient in strength or coördination, A the stress is carried still further backward, and the body wall itself strives to make up for the insufficiency of the extremity to the demand made upon it. In this manner may be produced abdominal incompetence, visceral ptosis and deformities of the spine, pelvis, thorax, and even of the other extremities, since insufficiency of the body wall may be further conveyed to these.

Examples of this will be given later. It is mentioned here because it is a manifestation of a law which seems to explain the action which insufficiently developed (in strength or coördination) extremities, both upper and lower, but especially the lower, have in causing visceral ptosis, this being one of the most common causes of this affection.

The converse of this is also observed. The circle of insufficiency and deformity may begin with abdominal incompetence or visceral ptosis, causing inefficiency of the trunk as a point of departure for developing activities of the extremities, leading to inefficiency or imperfect development of these extremities.

Those portions of the body wall which make up the abdomen are easily and early involved in attempts to compensate for weakness of the extremities.

If the abdominal walls have developed competence, they aid the body wall in meeting these demands, and the stress is met.

If the abdominal walls have not developed competence, they share in the stress, and are, by so much, diverted from their function of supporting and retaining the viscera. In this way, varying degrees of visceral ptosis are due to weakness of the extremities or of other portions of the body wall. The converse of this is also observed, as already noted.

EFFECT OF ABDOMINAL INCOMPETENCE ON THE MECHANICS OF RESPIRATION.

Clinical observation shows two main types of respiration:

(1) This may be called the anterior type. The abdomen is incompetent; the diaphragm and inspiring lungs make marked downward and forward excursion into the abdomen, displacing 